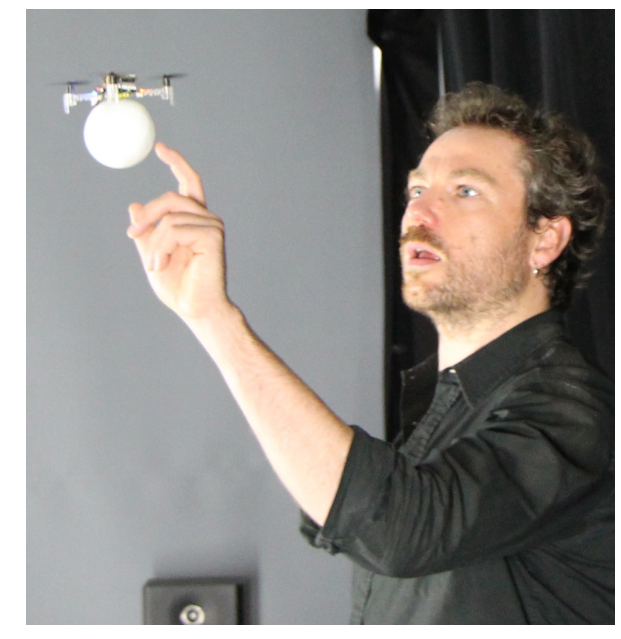

Figure 1: A juggler interacting with a flying ball

\section{Human-Drones Interaction for Gravity-Free Juggling}

Maxime Agor

Inria Bordeaux

Talence 33405, FR

Maxime.Agor@inria.fr

Damien Clergeaud

Inria Bordeaux

Talence 33405, FR
Damien.Clergeaud@inria.fr

\author{
Antoine Clée \\ Le cirque inachevé \\ Lille 62130, FR \\ cirque.inacheve@gmail.com
}

\section{Martin Hachet}

Inria Bordeaux

Talence 33405, FR

Martin.Hachet@inria.fr

\section{ABSTRACT}

In this work, we explore a new form of juggling. The juggler interacts with balls carried with nanodrones using $3 \mathrm{D}$ gestures. In this paper, we discuss technological as well as interaction issues.

\section{CCS CONCEPTS}

- Human-centered computing $\rightarrow$ Mixed / augmented reality; Interaction devices; Gestural input;

Permission to make digital or hard copies of part or all of this work for personal or classroom use is granted without fee provided that copies are not made or distributed for profit or commercial advantage and that copies bear this notice and the full citation on the first page. Copyrights for third-party components of this work must be honored. For all other uses, contact the Owner/Author.

IHM '17, August 29-September 1, 2017, Poitiers, France

( 2017 Copyright is held by the owner/author(s).

ACM ISBN 978-1-4503-5109-6/17/08. https://doi.org/10.1145/3132129.3132163 


\section{KEYWORDS}

Human-Drones Interaction, 3D gestures, Juggling, art.

\section{ACM Reference Format :}

M. Agor, A. Clée, D. Clergeaud and M. Hachet 2017. Human-Drones Interaction for Gravity-Free Juggling. In Proceedings of 29ème Conférence Francophone sur l'Interaction Homme-Machine, Poitiers Futuroscope, FR Août 2017 (IHM 2017), 6 pages.

DOI : $10.1145 / 3132129.3132163$

\section{INTRODUCTION}

Juggling is the art of manipulating objects. It is inherently linked to gravity. In this work, we explore an approach where we overpass this physical constraint, in order for an artist to explore new forms of interaction during a juggling performance.

In order to achieve this goal, we attached polystyrene balls to nano-quadcopters, in order to "defy laws of gravity", and to achieve otherwise impossible trajectories such as hovering, making circles, juggling in an horizontal plane, and possibly way more than that.

This approach, though, brings in a number of challenges, in broad topics going from drone control and localization to interaction techniques. In this paper, we will focus on the latter and explain our current investigations and first results.

\section{STATE OF THE ART}

\subsection{Art and science}

Connecting art and science has been at the heart of the motivation of many projects. An emblematic example is the work conducted by Merce Cunningham and the Simon Fraser University where they jointly created an interactive system supporting choreography. Their system, LifeForm, [6] [14] allowed the generation of sequences of movements that were visualized on a virtual skeleton. No on-scene representations of this dancing skeleton have been made at the time, though.

The music field is very innovative too when it comes to creating new interactions to improve their on-scene performances, especially since electronic-based instruments exist (see e.g. the work published at the NIME conference [8]). One famous example is the Léon Theremin's eponym instrument [16], which allowed the artist to play music by moving his hands in the air, without touching his instrument.

Closer to the juggling world, the jugglers-dancers company "Parabole" [2] worked on interactions with improved juggling material (such as infrared emitting balls) and synaesthetic spectacles, using light and sound effects correlated with the artist's or ball's movements. The Adrien M. and Claire B. 
company[1] also explores new forms of juggling. Eye-balls is another example of enhanced juggling by Marshall et al. [9].

Beside art performances, authors have also explored Human-drones interaction in various context.

\subsection{Human-drone interaction}

Jun Reikimoto and his team from the University of Tokyo experimented usage of a drone-powered ball in sports, such as football [10] or any hand-played sport [11]. Using a drone, in this case, allow them to equilibrate the game and make it enjoyable for everyone by changing the ball mechanics. This setup could also be used to create new sports, using non-conventional physics laws in a non-virtual environment. In order to interact with the ball, visual movement recognition is used, allowing, for example, to "throw" it without touching the ball, or to make a boomerang effect.

Another example is Drone \& Me [7], from the Stanford University, where they did a user study, exploring more than 400 interaction methods to see which one were considered the most intuitive by people. The results indicates that gestures and voice are usually preferred for Human-drones interaction.

Another team published a user study about this topic, called "How would you gesture navigate a drone?" [12], which focuses one gestural control, using either one or two hands.

\section{SYSTEM DESCRIPTION}

In this section, we will describe the system we have developed to interact with "gravity-free" balls.

The interaction process of this system is composed of three main parts; Human gesture, Trajectory generation, and Drone control. We describe these three components in the following sections.

\subsection{Human gesture}

Discussions with the juggler led to a set of requirements the system should have, inspired from standard juggling mechanics :

- The artists hands should be free, in order to keep using conventional objects too.

- Each hand had to be independent.

- The juggler would controls balls independently in order to make a global figure.

In order to retrieve the user's gestures to send commands, we use electronic gloves, with sensors embedded, allowing us to get the rotation and acceleration of the hand, and every finger motions. Using gloves also allows to keep standard juggling with conventional objects possible. Another way to retrieve the gesture information could have been $3 \mathrm{D}$ video tracking, but it brings too many problems on big tracking volumes. 


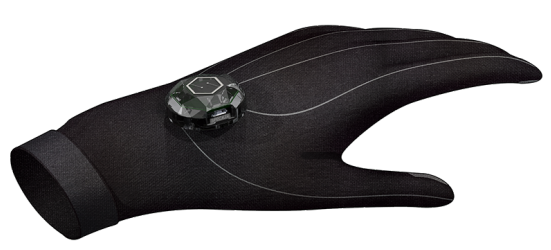

Figure 2: Specktr Pro glove

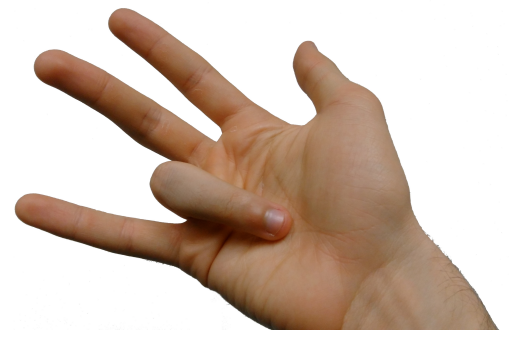

Figure 3: Selecting drone 4

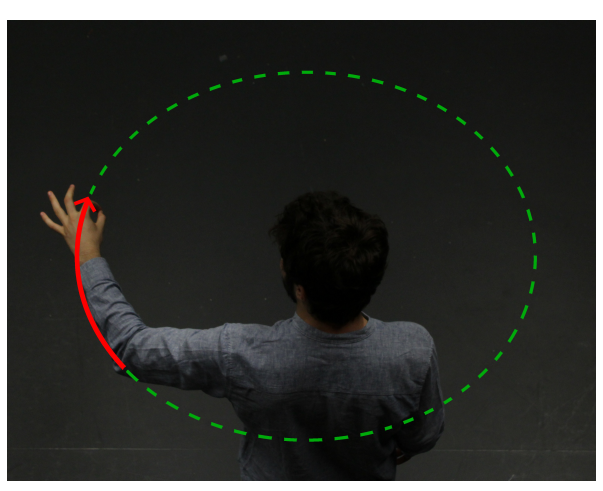

Figure 4: Circle and loop trajectory
We chose to use a pair of Specktr Pro [15] (See Figure 2), MIDI wireless gloves sold as a tool to make computer assisted music.

The juggler having to control multiple drones, with different types of trajectories, we had to design ways to select theses.

Two ways of selecting the drones have been investigated. The first one is to point at a drone. It has the advantage of being very visual to the public, but has several drawbacks too : it may be imprecise, and, more importantly, quickly alternating between drones in order to juggle may be complicated. The second way we thought of, and selected, is to select the drones by making a contact between a finger and the palm of the hand, where each finger correspond to a drone. (See Figure 3)

This method may also, in the future, allow the juggler to select multiple drones at once using multiple fingers.

Currently, we use a similar solution to select the command type. The juggler can select one of four commands by simply making a contact between his thumb and any finger.

While his fingers are touching, the juggler can make movements to "configure" the trajectory he wants to send to the drone. He can then release them to send the command.

\subsection{Trajectory generation}

In order to retrieve inputs from the glove, to compute and to send trajectories to the drones, we use a computer with the Robotic Operating System (ROS) [13] installed. ROS allows us to build an efficient and modular software, and to benefit from the whole robotics-community creations easily.

Once we retrieved the user gestures, we generate the drone's trajectory. There will be a large set of gestures and commands available. We started by implementing four of them.

First, we implemented the "Hover and Slide" command. It is the most basic one : It asks the drone to stay at its position, and allows, by quick slides in any direction, to change the position of the drone. Every other trajectories will be relative to the drone position.

The second trajectory is the "circle and loop". To use this one, the artist draws a portion of a circle in 3D (or even a full one), that we extrapolate to a full circle using geometric circle fitting algorithm [3], which allows the artist to modulate the radius by making a less-or-more curved movement (and to make a big circle without drawing the full one with its hand). Then we generate a trajectory following this circle. The drone will continue to follow it until it receives another command. (See Figure 4)

The third one allows the artist to draw an arbitrary shape that the drone will follow and loop onto To do so, we retrieve several waypoints from the artist hand movement and generate a closed b-spline curve passing through all of them.

The last one, and probably the most important, is the "ball movement" one. The juggler does a throwing gesture, as if he was throwing a real ball, and the drone will make a curve, as would a standard ball, by simulating gravity. The gravity direction, however, is relative to the rotation of the 


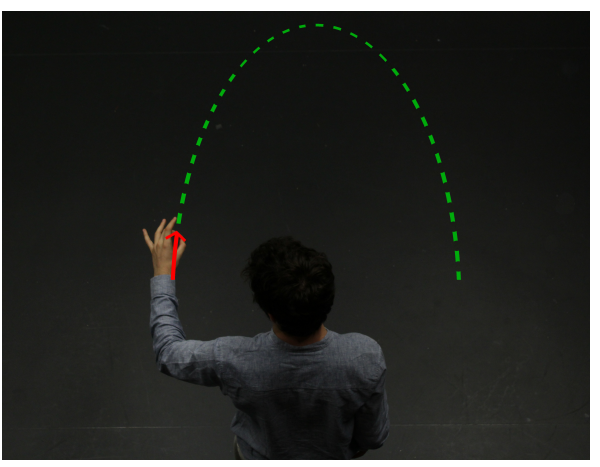

Figure 5: Ball movement trajectory

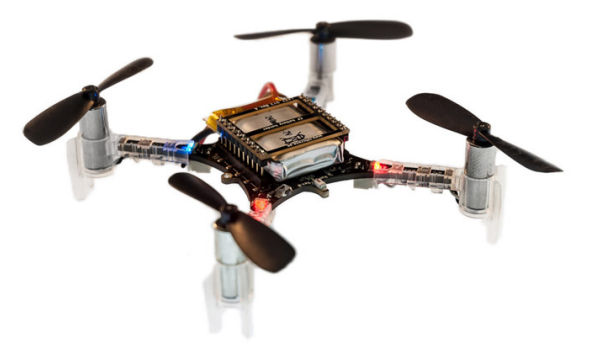

Figure 6: Crazyflie2 drone juggler movement around the axis X. This way, if he does a vertical movement, such as a traditional juggling throw, the drone will act as a standard ball, but an horizontal throw, for example, will make the drone follow an horizontal curve. (See Figure 5) This way, the artist can, by alternating this trajectory over multiple drones, juggle with them on any arbitrary axis.

\subsection{Drone control}

Once the trajectory is computed, we need to send commands to the drone.

We needed our drones to be roughly the same size as the balls, easily configurable, controllable with a computer, and with a way to know and control their absolute positions. We choose to use 5 crazyflie2 [4] (See Figure 6), an open-source nano-quadcopter sold by the company Bitcraze, and 8 loco positionning nodes [5], a rather cheap indoor localization system sold by the same manufacturer

We then attached polystyrene balls under them, as shown in Figure 1.

We control theses drones through a $2.4 \mathrm{GHz}$ radio, by sending them the positions we want them to go to, and each drone internally computes how to fly to the given position.

\section{CONCLUSION AND FUTURE IMPROVEMENTS}

We have developed a system that allows us to control and send commands to 5 drones from 4 different trajectories, using simple gestures. This work is currently in progress, and we are improving its reliability.

The next step of this work will consist in exploring the usability of the system with the artist. To do so, we will organize working sessions where we will refine our technological system based on the needs and requirements of the final user. A challenge will be to transfer the virtuosity of the juggler to a fine and precise control of the drones.

Following work may include more subtle commands, using for example the hand rotation to generate and control new expressive drones trajectories. The simultaneous selection of multiple drones will be part of our future work, too. We will also continue working on more technical issues, such as securities for trajectory crossings, and precise trajectory tracking, with speed modulations.

The user experience of the audience facing such a gravity-free juggling performance, as well as the level of virtuosity of the juggler is also something to explore. Dedicated user studies should be planned for this purpose.

\section{RÉFÉRENCES}

[1] [n. d.]. AdrienM and Claire B. ([n. d.]). http://www.am-ch.net/en/a-propos

[2] [n. d.]. Parabole. ([n. d.]). http://www.cieparabole.com 
[3] Sung Joon Ahn, Wolfgang Rauh, and Hans-JÃijrgen Warnecke. 2001. Least-squares orthogonal distances fitting of circle, sphere, ellipse, hyperbola, and parabola. Pattern Recognition 34, 12 (2001), 2283 - 2303. https://doi.org/10.1016/ S0031-3203(00)00152-7

[4] Bitcraze. 2014. Crazyflie2, an open source nano-quadcopter. https://www.bitcraze.io/crazyflie-2/. (2014).

[5] Bitcraze. 2016. Loco Positioning System : An indoor localization system. https://www.bitcraze.io/loco-pos-system/. (2016).

[6] Tom W. Calvert, Armin Bruderlin, Sang Mah, Thecla Schiphorst, and Chris Welman. 1993. The Evolution of an Interface for Choreographers. In Proceedings of the INTERACT' 93 and CHI '93 Conference on Human Factors in Computing Systems (CHI '93). ACM, New York, NY, USA, 115-122. https://doi.org/10.1145/169059.169113

[7] Jessica R. Cauchard, Jane L. E, Kevin Y. Zhai, and James A. Landay. 2015. Drone \&\#38; Me : An Exploration into Natura Human-drone Interaction. In Proceedings of the 2015 ACM International Joint Conference on Pervasive and Ubiquitous Computing (UbiComp '15). ACM, New York, NY, USA, 361-365. https://doi.org/10.1145/2750858.2805823

[8] Alexander Refsum Jensenius and Michael Lyons (Eds.). 2017. Augmenting Instruments and Extending Cultures : on the Overtone Violin (1 ed.). Springer International Publishing.

[9] Joe Marshall, Steve Benford, and Tony Pridmore. 2007. Eye-balls : Juggling with the Virtual. In Proceedings of the 6th ACM SIGCHI Conference on Creativity \&Amp; Cognition (C\&C '07). ACM, New York, NY, USA, 265-266. https: //doi.org/10.1145/1254960.1255005

[10] Kei Nitta, Keita Higuchi, and Jun Rekimoto. 2014. HoverBall : Augmented Sports with a Flying Ball. In Proceedings of the 5th Augmented Human International Conference (AH '14). ACM, New York, NY, USA, Article 13, 4 pages. https: //doi.org/10.1145/2582051.2582064

[11] Kei Nitta, Keita Higuchi, Yuichi Tadokoro, and Jun Rekimoto. 2015. Shepherd Pass : Ability Tuning for Augmented Sports Using Ball-shaped Quadcopter. In Proceedings of the 12th International Conference on Advances in Computer Entertainment Technology (ACE '15). ACM, New York, NY, USA, Article 11, 7 pages. https://doi.org/10.1145/2832932.2832950

[12] Mohammad Obaid, Felix Kistler, Gabrielè Kasparavičiūté, Asim Evren Yantaç, and Morten Fjeld. 2016. How Would You Gesture Navigate a Drone ?: A User-centered Approach to Control a Drone. In Proceedings of the 20th International Academic Mindtrek Conference (AcademicMindtrek '16). ACM, New York, NY, USA, 113-121. https://doi.org/10.1145/2994310.2994348

[13] Morgan Quigley, Ken Conley, Brian Gerkey, Josh Faust, Tully Foote, Jeremy Leibs, Rob Wheeler, and Andrew Y Ng. 2009. ROS : an open-source Robot Operating System. In ICRA workshop on open source software, Vol. 3. Kobe, 5.

[14] Thecla Schiphorst. 1993. A case study of Merce CunninghamâĂŹs use of the lifeforms computer choreographic system in the making of trackers. Ph.D. Dissertation. Simon Fraser University.

[15] Specktr. 2016. Specktr Pro, MIDI gloves for MAO. http://specktr.com/fr/specktr-pro/. (2016)

[16] Leon S Theremin and Oleg Petrishev. 1996. The design of a musical instrument based on cathode relays. Leonardo Music ournal 6, 1 (1996), 49-50. 